\title{
Option Pricing under Risk-Minimization Criterion in an Incomplete Market with the Finite Difference Method
}

\author{
Xinfeng Ruan, ${ }^{1}$ Wenli Zhu, ${ }^{1}$ Shuang Li, $^{2}$ and Jiexiang Huang ${ }^{1}$ \\ ${ }^{1}$ School of Economic Mathematics, Southwestern University of Finance and Economics, Chengdu 611130, China \\ ${ }^{2}$ Department of Mathematics and Statistics, Curtin University, Perth, WA 6102, Australia \\ Correspondence should be addressed to Xinfeng Ruan; ruanxinf@gmail.com
}

Received 16 January 2013; Revised 22 March 2013; Accepted 13 April 2013

Academic Editor: Guangchen Wang

Copyright (c) 2013 Xinfeng Ruan et al. This is an open access article distributed under the Creative Commons Attribution License, which permits unrestricted use, distribution, and reproduction in any medium, provided the original work is properly cited.

\begin{abstract}
We study option pricing with risk-minimization criterion in an incomplete market where the dynamics of the risky underlying asset is governed by a jump diffusion equation with stochastic volatility. We obtain the Radon-Nikodym derivative for the minimal martingale measure and a partial integro-differential equation (PIDE) of European option. The finite difference method is employed to compute the European option valuation of PIDE.
\end{abstract}

\section{Introduction}

Option pricing problem is one of the predominant concerns in the financial market. Since the advent of the justly celebrated Black-Scholes option pricing formula in [1], there has been an increasing amount of the literature describing the theory and its practice. In the Black-Scholes model, the appreciation rate and the volatility rate are assumed to be constants. However, more and more empirical evidence has revealed that these assumptions are not consistent with reality. Hence, many option valuation models which relax some of the restrictive assumptions in the Black-Scholes framework have been proposed and tested, such as the stochastic volatility models in [2-8], the jump diffusion or the Lévy process models in [9-12]. The model to be studied in this paper takes into account not only stochastic volatility based on Heston's model in [8], but also the jump diffusion case. Therefore, several new problems and difficulties are exposed in our model.

Different from the Black-Scholes framework which uses jump diffusion to describe the price dynamics of underlying asset, the market of our model is incomplete; that is, it is not possible to replicate the payoff of every contingent claim by a portfolio, and there are several equivalent martingale measures. How to choose a consistent pricing measure from the set of equivalent martingale measures becomes an important problem. This means that we need to find some criterions to determine one from the set of equivalent martingale measures in some economically or mathematically motivated fashion. Föllmer and Leukert (2000), Kallsen (1999), Cvitanić et al. (2001), and Bielecki and Jeanblanc (2008) in [13-16] identified a unique equivalent martingale measure by utility maximization. Then, the option valuation under the minimal martingale measure was further developed by several researchers. Schweizer (1991) and Föllmer and Schweizer (1991) in $[17,18]$ found that under the minimal martingale measure, a unique risk-minimizing (or optimal) strategy hedging of contingent claims in incomplete market exists. In our paper, we name the criterion under the minimal martingale measure as a risk-minimization criterion. Thus, in the incomplete market, option pricing is approximately possible with risk-minimization criterion. As presented in this paper, our work is based on the task of Föllmer and Schweizer in $[17,18]$, and the purpose of this paper is to find the minimal martingale measure and the measure switch of asset prices processes with stochastic volatility and jump diffusion. By employing the minimal martingale measure, we obtain the Radon-Nikodym derivative and a partial integrodifferential equation (PIDE) for the European option.

However, it is difficult to get the exact solution of the PIDE in our model. Several numerical methods have been proposed to solve the PIDE approximately. The methods 
include numerical integration by Chiarella and Ziogas (2009) in [19], finite elements by Matache et al. (2005) in [20], the method of lines by Meyer (1998) in [21], and the finite difference methods (FDM) including those by Carr and Hirsa (2003) in [22], d'Halluin et al. (2004) in [23], and Briani et al. (2004) in [24]. This paper employs the FDM to compute the valuation of European option, and American option. Then further studies on using other methods to compute the solution of PIDE and derive the pricing formula of another option will be included in our future study.

The rest of the paper is organized as follows. In Section 2, we present the model for the underlying market and DoobMeyer decomposition of the risky asset. In Section 3, we investigate an explicit representation of the density process of the minimal martingale measure. In Section 4, we derive a PIDE for the European option. The European option pricing with FDM is then studied in Section 5. Numerical results are shown in Section 6, and conclusions are given in Section 7.

\section{The Model}

In this paper, we consider the financial market with the following two basic assets:

(i) a Bond whose price $B_{t}$ at time $t$ is given by

$$
d B_{t}=r_{t} B_{t} d t, \quad B_{0}=1
$$

(ii) a Stock whose price $S_{t}$ at time $t$ is given by

$$
\begin{gathered}
\frac{d S_{t}}{S_{t^{-}}}=\mu_{t} d t+\sqrt{V_{t}} d W_{t}^{S}+\int_{R_{0}}(y-1) \bar{N}(d u, d y), \quad S_{0}>0, \\
d V_{t}=\kappa\left(\varphi-V_{t}\right) d t+\sigma \sqrt{V_{t}} d W_{t}^{V}, \quad V_{0}>0 .
\end{gathered}
$$

Here, $t \in[0, T], y \in R_{0} \subset \mathbb{R} /\{0\}$, and $T>0$; on the filtered complete space $\left(\Omega, \mathscr{F},\left\{\mathscr{F}_{t}\right\}_{t \geq 0}, P\right)$, there are $W_{t}^{S}, W_{t}^{V}$ which both are 1-dimensional Brownian motions with $d W_{t}^{S} d W_{t}^{V}=\rho d t$ and $\bar{N}(d t, d y)=N(d t, d y)-v(d y) d t$ which is the compensated jump measure of $\eta(\cdot) . N(d t, d y)$ is the jump measure, and $v(d y)$ is the Lévy measure of the Lévy process $\eta(\cdot) . \eta(t)$ is given by $\eta(t)=\int_{0}^{t} \int_{R_{0}}(y-1) \bar{N}(d u, d y)$, $t \geq 0$. Additionally, $\int_{R_{0}}(y-1)^{2} v(d y)<\infty$.

From above settings, the risk is at least two dimensional including the Brownian motion and Lévy process but only one risky asset in the market. It is impossible to exactly replicate the payoff of a given option by a dynamic portfolio strategy of the basic assets which is self-financing. We all know that if the dynamic portfolio strategy exists, then the initial cost of the portfolio must equal the price of the option, and the market is called complete; otherwise, an arbitrage opportunity exists, and the market is called incomplete. Therefore, the market of our model is incomplete since the perfect replication is impossible.

Compared with the Black-Scholes model, our model remedies some serious drawbacks of the Black-Scholes model, such as the constant volatility assumption and market completeness assumption. Bakshi et al. in [10] systematically analyzed the performance of the stochastic volatility model, the jump diffusion model, and the stochastic interest rate model, and concluded that a model with stochastic volatility and the jump diffusion was a better alternative to the BlackScholes model, because the former not only performed far better but also was practically implementable. An excellent innovation of our model is combining stochastic volatility model and the jump diffusion model. Consequently, our model is better than the Black-Scholes model to portray realistic financial markets in theory and practice.

Now, we want to find a unique optimal strategy hedging of contingent claims under risk-minimization criterion. Based on [18], it is equivalent to find the minimal martingale measure from the set of equivalent martingale measures and then obtain approximate prices of contingent claims.

With the Doob-Meyer decomposition, the discounted risky asset price process, $\widehat{S}_{t}=e^{-\int_{0}^{t} r_{s} d s} S_{t}$, is a special semimartingale and can be written as

$$
\widehat{S}_{t}=\widehat{S}_{0}+M_{t}+A_{t}
$$

with

$$
\begin{gathered}
M_{t}=\int_{0}^{t} \widehat{S}_{u^{-}} \sqrt{V_{u}} d W_{u}^{S}+\int_{0}^{t} \int_{R_{0}} \widehat{S}_{u^{-}} y \bar{N}(d u, d y), \\
A_{t}=\int_{0}^{t} \widehat{S}_{u^{-}}\left(\mu_{u}-r_{u}\right) d u
\end{gathered}
$$

where $M_{t}$ is the martingale part of $\widehat{S}_{t}$ and $A_{t}$ is the predictable process of finite variation.

\section{Minimal Martingale Measure}

We introduce the nations of minimal martingale measure in this section. Föllmer and Schweizer (1991) [18] noticed that the optimal hedging strategy can be computed in terms of the minimal martingale measure. Furthermore, it is uniquely determined. Hence, under the minimal martingale measure, the Radon-Nikodym derivative can be found and computed. Before that, we define the minimal martingale measure.

Definition 1 (see [18]). A local martingale measure $\widetilde{P}$, equivalent to the original measure $P$, is called minimal if $\widetilde{P}=P$ on $\mathscr{F}_{t}$ and if any square-integrable $P$-martingale $L$ which is $P$ orthogonal to $M$ remains a local martingale under $\widetilde{P}$.

Theorem 2 (see [18]). (i) The minimal martingale measure $\widetilde{P}$ is uniquely determined.

(ii) $\widetilde{P}$ exists if and only if there exists a predictable process $\beta_{t}$ that satisfies

$$
Z_{t}=\frac{d \widetilde{P}}{d P}=1+\int_{0}^{t} \beta_{t} d M_{t}
$$

Using Theorem 2, we obtain the following theorem for computing the Radon-Nikodym derivative. 
Theorem 3. The Radon-Nikodym derivative under the minimal martingale measure $\widetilde{P}$ is

$$
\begin{aligned}
Z_{t}=\exp \{ & -\int_{0}^{t} \theta_{t} \sqrt{V_{u}} d W_{u}^{S}-\frac{1}{2} \int_{0}^{t} \theta_{u}^{2} V_{u} d u \\
& +\int_{0}^{t} \int_{R_{0}} \ln \left(1-\theta_{u}(y-1)\right) N(d u, d y) \\
& \left.+\int_{0}^{t} \int_{R_{0}} \theta_{u}(y-1) v(d y) d u\right\} .
\end{aligned}
$$

Proof. The theory of the Girsanov transformation shows that the predictable process of bounded variation can also be computed in terms of $Z_{t}$ :

$$
-d A_{t}=\frac{1}{Z_{t-}} d\langle M, Z\rangle_{t}
$$

Throughout this paper, we make use of the notations that $\langle A, B\rangle_{t}$ defined the quadratic variation process between $A$ and $B$ and denote $\langle A\rangle_{t} \doteq\langle A, A\rangle_{t}$. Under $\widetilde{P}$, the predictable process of bounded variation in the Doob-Meyer decomposition of $M$ is given by

$$
\frac{1}{Z_{t-}} d\langle M, Z\rangle_{t}=\frac{1}{Z_{t-}} d\langle M\rangle_{t}=-d A_{t} .
$$

Using (6) and (9), we have

$$
Z_{t}=1-\int_{0}^{t} Z_{u-} \frac{d A_{u}}{d\langle M\rangle_{u}} d M_{u}
$$

Denote $d Y_{u}=-\left(d A_{u} / d\langle M\rangle_{u}\right) d M_{u}$, then (10) can be written as

$$
Z_{t}=1+\int_{0}^{t} Z_{u_{-}} d Y_{u}
$$

From (4), we get

$$
\begin{aligned}
\langle M\rangle_{t}= & \left\langle\int_{0}^{t} \widehat{S}_{u^{-}} \sqrt{V_{u}} d W_{u}^{S}\right. \\
& \left.\quad+\int_{0}^{t} \int_{R_{0}} \widehat{S}_{u^{-}}(y-1) \bar{N}(d u, d y)\right\rangle \\
= & \int_{0}^{t} \widehat{S}_{u^{-}}^{2}\left(\sqrt{V_{u}}\right)^{2} d u \\
& +\int_{0}^{t} \int_{R_{0}} \widehat{S}_{u^{-}}^{2}(y-1)^{2} v(d y) d u \\
= & \int_{0}^{t} \widehat{S}_{u^{-}}^{2}\left(V_{u}+\int_{R_{0}}(y-1)^{2} v(d y)\right) d u .
\end{aligned}
$$

Hence,

$$
\begin{aligned}
d Y_{u}= & -\frac{d A_{u}}{d\langle M\rangle_{u}} d M_{u} \\
= & -\frac{\widehat{S}_{u^{-}}\left(\mu_{u}-r_{u}\right) d u}{\widehat{S}_{u^{-}}^{2}\left(v_{u}+\int_{R_{0}}(y-1)^{2} v(d y)\right) d u} \\
& \times\left[\widehat{S}_{u^{-}}\left(\sqrt{V_{u}} d W_{u}^{S}+\int_{R_{0}} y \bar{N}(d u, d y)\right)\right] \\
= & -\frac{\left(\mu_{u}-r_{u}\right)\left(\sqrt{V_{u}} d W_{u}^{S}+\int_{R_{0}}(y-1) \bar{N}(d u, d y)\right)}{V_{u}+\int_{R_{0}}(y-1)^{2} v(d y)} .
\end{aligned}
$$

From (11), we know that $Z_{t}$ is the Doléans-Dade exponential. Thus, we obtain

$$
\begin{gathered}
Z_{t}=1+\int_{0}^{t} Z_{u^{-}} d Y_{u}, \quad Z_{0}=1, \\
d Y_{u}=-\theta_{u}\left(\sqrt{V_{u}} d W_{u}^{S}+\int_{R_{0}}(y-1) \bar{N}(d u, d y)\right),
\end{gathered}
$$

where

$$
\theta_{u}=\frac{\left(\mu_{u}-r_{u}\right)}{V_{u}+\int_{R_{0}}(y-1)^{2} v(d y)} .
$$

Solving (14), we obtain $Z_{t}$ in Theorem 3.

Remark 4. The Brown motions under the minimal martingale measure $\widetilde{P}$ are

$$
\begin{gathered}
\widetilde{W}_{t}^{S}=W_{t}^{S}+\int_{0}^{t} \theta_{t} \sqrt{V_{u}} d u, \\
\widetilde{W}_{t}^{V}=W_{t}^{V}+\rho \int_{0}^{t} \theta_{t} \sqrt{V_{u}} d u,
\end{gathered}
$$

and the compensatory of $N(d u, d y)$ is

$$
\begin{gathered}
\widetilde{v}(d y) d u=\left(1-\theta_{u}(y-1)\right) v(d y) d u, \\
\widetilde{N}(d u, d y)=N(d u, d y)-\widetilde{v}(d y) d u .
\end{gathered}
$$

Remark 5. Equation (2) under the minimal martingale measure $\widetilde{P}$ is written as

$$
\begin{aligned}
\frac{d S_{t}}{S_{t^{-}}}= & \mu_{t} d t+\sqrt{V_{t}} d \widetilde{W}_{t}^{S} \\
& +\int_{R_{0}}(y-1) \widetilde{N}(d t, d y)-\theta_{t} V_{t} d t \\
& -\int_{R_{0}} \theta_{t}(y-1)^{2} v(d y) d t, \quad S_{0}>0, \\
d V_{t}=\kappa & \left(\varphi-V_{t}\right) d t+\sigma \sqrt{V_{t}} d \widetilde{W}_{t}^{V}-\rho \sigma \theta_{t} V_{t} d t .
\end{aligned}
$$

To guarantee that $\widehat{S}_{t}$ is a martingale under the minimal martingale measure $\widetilde{P}$, the following corollary is necessary. 
Corollary 6. Under the minimal martingale measure $\widetilde{P}, \widehat{S}_{t}$ is a martingale if and only if

$$
\mu_{t}-r_{t}-\theta_{t} V_{t}-\int_{R_{0}} \theta_{t}(y-1)^{2} v(d y)=0 .
$$

Proof. Substituting $S_{t}=e^{\int_{0}^{t} r_{s} d s} \widehat{S}_{t}$ into (18), since $\widehat{S}_{t}$ is a martingale, the drift term must be identical to zero. Then, we can get (19).

\section{Partial Integro-Differential Equation for European Call Option}

Under the minimal martingale measure $\widetilde{P}$, the price of the European call option $C\left(t, S_{t}, V_{t}\right)$ at time $t$ with strike price $K$ and maturity date $T$ is given by

$$
C\left(t, S_{t}, V_{t}\right)=\mathbb{E}^{\tilde{P}}\left[e^{-\int_{t}^{T} r_{u} d u}\left(S_{T}-K\right)^{+} \mid \mathscr{F}_{t}\right],
$$

and $C\left(T, S_{T}, V_{T}\right)=\left(S_{T}-K\right)^{+}$.

By the fact that the discounted price of the European call option is a martingale under $\widetilde{P}$, we can obtain the following theorem.

Theorem 7. The price of the European call option satisfies the following PIDE:

$$
\begin{aligned}
0= & -r_{t} C\left(t, S_{t}, V_{t}\right)+\frac{\partial C}{\partial t}+\frac{\partial C}{\partial S} r_{t} S_{t^{-}} \\
& +\frac{\partial C}{\partial V}\left(\kappa\left(\varphi-V_{t}\right)-\rho \sigma \theta_{t} V_{t}\right) \\
+ & \frac{1}{2} \frac{\partial^{2} C}{\partial S^{2}} V_{t} S_{t^{-}}^{2}+\frac{\partial^{2} C}{\partial S \partial V} S_{t^{-}} V_{t} \sigma \rho \\
+ & \frac{1}{2} \frac{\partial^{2} C}{\partial V^{2}} V_{t} \sigma^{2}+\int_{R_{0}}\left(C\left(t, S_{t^{-}} y, V_{t^{-}}\right)-C\left(t, S_{t^{-}}, V_{t^{-}}\right)\right. \\
& \left.\quad-(y-1) \frac{\partial C}{\partial S} S_{t^{-}}\right) \widetilde{v}(d y),
\end{aligned}
$$

and $C\left(T, S_{T}, V_{T}\right)=\left(S_{T}-K\right)^{+}$.

Proof. The total derivative of the discounted option price is

$$
\begin{aligned}
d\left(e^{-\int_{0}^{t} r_{u} d u} C\left(t, S_{t}, V_{t}\right)\right) \\
=-r_{t} e^{-\int_{0}^{t} r_{u} d u} C\left(t, S_{t}, V_{t}\right) d t \\
\quad+e^{-\int_{0}^{t} r_{u} d u} \frac{\partial C}{\partial t} d t+e^{-\int_{0}^{t} r_{u} d u} \frac{\partial C}{\partial S} d S^{C} \\
\quad+e^{-\int_{0}^{t} r_{u} d u} \frac{\partial C}{\partial V} d V^{C}+\frac{1}{2} e^{-\int_{0}^{t} r_{u} d u} \frac{\partial^{2} C}{\partial S^{2}} d S^{C} d S^{C} \\
+e^{-\int_{0}^{t} r_{u} d u} \frac{\partial^{2} C}{\partial S \partial V} d S^{C} d V^{C}+\frac{1}{2} e^{-\int_{0}^{t} r_{u} d u} \frac{\partial^{2} C}{\partial V^{2}} d V^{C} d V^{C} \\
\quad+e^{-\int_{0}^{t} r_{u} d u} \int_{R_{0}}\left(C\left(t, S_{t^{-}} y, V_{t^{-}}\right)\right.
\end{aligned}
$$$$
\left.-C\left(t, S_{t^{-}}, V_{t^{-}}\right)\right) N(d t, d y)
$$$$
=e^{-\int_{0}^{t} r_{u} d u}\left\{-r_{t} C\left(t, S_{t}, V_{t}\right) d t+\frac{\partial C}{\partial t} d t\right.
$$$$
+\frac{\partial C}{\partial S} S_{t^{-}}\left(\mu_{t} d t+\sqrt{V_{t}} d \widetilde{W}_{t}^{S}-\theta_{t} V_{t} d t\right.
$$$$
-\int_{R_{0}} \theta_{t}(y-1)^{2} v(d y) d t
$$$$
\left.-\int_{R_{0}}(y-1) \widetilde{v}(d y) d t\right)
$$$$
+\frac{\partial C}{\partial V}\left(\kappa\left(\varphi-V_{t}\right) d t+\sigma \sqrt{V_{t}} d \widetilde{W}_{t}^{V}\right.
$$$$
\left.-\rho \sigma \theta_{t} V_{t} d t\right)
$$$$
+\frac{1}{2} \frac{\partial^{2} C}{\partial S^{2}} V_{t} S_{t^{-}}^{2} d t+\frac{\partial^{2} C}{\partial S \partial V} S_{t^{-}} V_{t} \sigma \rho d t
$$$$
+\frac{1}{2} \frac{\partial^{2} C}{\partial V^{2}} V_{t} \sigma^{2} d t
$$$$
+\int_{R_{0}}\left(C\left(t, S_{t^{-}} y, V_{t^{-}}\right)\right.
$$$$
\left.-C\left(t, S_{t^{-}}, V_{t^{-}}\right)\right) \widetilde{N}(d t, d y)
$$$$
+\int_{R_{0}}\left(C\left(t, S_{t^{-}} y, V_{t^{-}}\right)\right.
$$$$
\left.\left.-C\left(t, S_{t^{-}}, V_{t^{-}}\right)\right) \widetilde{v}(d y) d t\right\}
$$$$
=e^{-\int_{0}^{t} r_{u} d u}\left\{-r_{t} C\left(t, S_{t}, V_{t}\right)+\frac{\partial C}{\partial t}+\frac{\partial C}{\partial S} r_{t} S_{t^{-}}\right.
$$$$
+\frac{\partial C}{\partial V}\left(\kappa\left(\varphi-V_{t}\right)-\rho \sigma \theta_{t} V_{t}\right)
$$$$
+\frac{1}{2} \frac{\partial^{2} C}{\partial S^{2}} V_{t} S_{t^{-}}^{2}+\frac{\partial^{2} C}{\partial S \partial V} S_{t^{-}} V_{t} \sigma \rho
$$$$
+\frac{1}{2} \frac{\partial^{2} C}{\partial V^{2}} V_{t} \sigma^{2}
$$$$
+\int_{R_{0}}\left(C\left(t, S_{t^{-}} y, V_{t^{-}}\right)\right.
$$$$
-C\left(t, S_{t^{-}}, V_{t^{-}}\right)
$$$$
\left.\left.-(y-1) \frac{\partial C}{\partial S} S_{t^{-}}\right) \widetilde{v}(d y)\right\} d t
$$$$
+e^{-\int_{0}^{t} r_{u} d u}\left\{\frac{\partial C}{\partial S} S_{t^{-}} \sqrt{v_{t}} d \widetilde{W}_{t}^{S}+\frac{\partial C}{\partial V} \sigma \sqrt{V_{t}} d \widetilde{W}_{t}^{V}\right.
$$$$
+\int_{R_{0}}\left(C\left(t, S_{t^{-}} y, V_{t^{-}}\right)\right.
$$$$
\left.\left.-C\left(t, S_{t^{-}}, V_{t^{-}}\right)\right) \widetilde{N}(d t, d y)\right\} .
$$ 
We make the drift term zero, since the discounted price of the European put option is a martingale. Then, we obtain in Theorem 7.

\section{Finite Difference Scheme of European Call Option}

5.1. The Problem. In this section, we will derive the finite difference scheme for PIDE (21). Before that, we assume that $r_{t}=r, \mu_{t}=\mu, R_{0}=[0, \infty]$ and $\nu(d y) d t=\lambda f(y) d y d t$. In this paper, we take Merton's jump diffusion model with the density $f(y)$ which is from the log-normal distribution:

$$
f(y)=\frac{1}{y \delta \sqrt{2 \pi}} \exp \left(-\frac{(\ln y-\gamma)^{2}}{2 \delta^{2}}\right) .
$$

Furthermore, using error function, we denote that

$$
\begin{gathered}
K_{0}:=\int_{R_{0}} v(d y)=1, \\
K_{1}:=\int_{R_{0}}(y-1) v(d y)=e^{\left(\delta^{2} / 2\right)+\gamma}-1, \\
K_{2}:=\int_{R_{0}}(y-1)^{2} v(d y) \\
=\frac{1}{\sqrt{1-2 \delta^{2}}} e^{\gamma^{2} /\left(1-2 \delta^{2}\right)}-2 e^{\left(\delta^{2} / 2\right)+\gamma}+1, \\
\theta_{u}:=\frac{\mu-r}{V_{u}+\int_{R_{0}}(y-1)^{2} v(d y)}=\frac{\mu-r}{V_{u}+K_{2}},
\end{gathered}
$$

where $r, \mu, \lambda, \delta, \gamma, K_{0}, K_{1}$, and $K_{2}$ are constants, and $\delta^{2}<$ $1 / 2$.

From (21) and (19), we get the PIDE with original parameters in the original model (2) as follows:

$$
\begin{aligned}
0= & -\left(r_{t}+K_{0}-\theta_{t} K_{1}\right) C\left(t, S_{t}, V_{t}\right) \\
& +\frac{\partial C}{\partial t}+\frac{\partial C}{\partial S}\left(r_{t}-K_{1}+\theta_{t} K_{2}\right) S_{t^{-}} \\
& +\frac{\partial C}{\partial V}\left(\kappa\left(\varphi-V_{t}\right)-\rho \sigma \theta_{t} V_{t}\right) \\
& +\frac{1}{2} \frac{\partial^{2} C}{\partial S^{2}} V_{t} S_{t^{-}}^{2}+\frac{\partial^{2} C}{\partial S \partial V} S_{t^{-}} V_{t} \sigma \rho+\frac{1}{2} \frac{\partial^{2} C}{\partial V^{2}} V_{t} \sigma^{2} \\
& +\int_{R_{0}} C\left(t, S_{t^{-}} y, V_{t^{-}}\right) v(d y) \\
& -\theta_{t} \int_{R_{0}} C\left(t, S_{t^{-}} y, V_{t^{-}}\right)(y-1) v(d y) .
\end{aligned}
$$

To represent conveniently, we denote that

$$
\begin{gathered}
\mathrm{I}(t, S, V):=\int_{0}^{\infty} C\left(t, S_{t^{-}}, V_{t^{-}}\right) f(y) d y, \\
\mathrm{II}(t, S, V):=\int_{0}^{\infty} C\left(t, S_{t^{-}}, V_{t^{-}}\right)(y-1) f(y) d y .
\end{gathered}
$$

Then, (25) can be written as

$$
\begin{aligned}
& 0=-\left(r+K_{0}-\theta K_{1}\right) C(t, S, V)+\frac{\partial C}{\partial t} \\
&+\frac{\partial C}{\partial S}\left(r-K_{1}+\theta K_{2}\right) S \\
&+ \frac{\partial C}{\partial V}(\kappa(\varphi-V)-\rho \sigma \theta V) \\
&+ \frac{1}{2} \frac{\partial^{2} C}{\partial S^{2}} V S^{2}+\frac{\partial^{2} C}{\partial S \partial V} S V \sigma \rho \\
&+ \frac{1}{2} \frac{\partial^{2} C}{\partial V^{2}} V \sigma^{2}+\lambda \mathrm{I}(t, S, V)-\lambda \theta \mathrm{II}(t, S, V), \\
& C(T, S, V)=(S-K)^{+}, \\
& C C(t, 0, V)=0, \\
& \lim _{\rightarrow \infty} C(t, S, V)=\left(S \max _{-}-K\right) e^{-r(T-t)}, \\
&+\left(r+K_{0}-\frac{(\mu-r) K_{1}}{K_{2}}\right) C(t, S, 0) \\
&+\frac{\partial C}{\partial t}+\frac{\partial C}{\partial S}\left(r-K_{1}+(\mu-r)\right) S \\
&+\frac{\partial C}{\partial V} \kappa \varphi+\lambda \mathrm{I}(t, S, 0)-\lambda \frac{(\mu-r)}{K_{2}} \mathrm{II}(t, S, 0) .
\end{aligned}
$$

Now, we denote $\tau:=T-t, \widetilde{C}(\tau, S, V):=C(T-\tau, S$, $V)=C(t, S, V), \widetilde{\mathrm{I}}(t, S, V):=\mathrm{I}(T-\tau, S, V)=\mathrm{I}(t, S, V)$, and $\widetilde{\mathrm{II}}(t, S, V):=\operatorname{II}(T-\tau, S, V)=\mathrm{II}(t, S, V)$. Then we have the following problem equivalent to (27).

Problem 1. Consider

$$
\begin{aligned}
& \frac{\partial \widetilde{C}}{\partial \tau}=-\left(r+K_{0}-\theta K_{1}\right) \widetilde{C}(\tau, S, V) \\
&+ \frac{\partial \widetilde{C}}{\partial S}\left(r-K_{1}+\theta K_{2}\right) S \\
&+ \frac{\partial \widetilde{C}}{\partial V}(\kappa(\varphi-V)-\rho \sigma \theta V)+\frac{1}{2} \frac{\partial^{2} \widetilde{C}}{\partial S^{2}} V S^{2} \\
&+ \frac{\partial^{2} \widetilde{C}}{\partial S \partial V} S V \sigma \rho+\frac{1}{2} \frac{\partial^{2} \widetilde{C}}{\partial V^{2}} V \sigma^{2} \\
&+ \lambda \widetilde{\mathrm{I}}(\tau, S, V)-\lambda \theta \widetilde{\mathrm{I}}(\tau, S, V), \\
& \widetilde{C}(0, S, V)=(S-K)^{+}, \\
& \widetilde{C}(\tau, 0, V)=0, \\
& \lim _{S \rightarrow \infty} \widetilde{C}(\tau, S, V)=\left(S_{\max }-K\right) e^{-r \tau}, \\
& \lim _{V \rightarrow \infty} \widetilde{C}(\tau, S, V)=S,
\end{aligned}
$$




$$
\begin{aligned}
0= & -\left(r+K_{0}-\frac{(\mu-r) K_{1}}{K_{2}}\right) \widetilde{C}(\tau, S, 0) \\
& +\frac{\partial \widetilde{C}}{\partial \tau}+\frac{\partial \widetilde{C}}{\partial S}\left(r-K_{1}+(\mu-r)\right) S \\
& +\frac{\partial \widetilde{C}}{\partial V} \kappa \varphi+\lambda \widetilde{\mathrm{I}}(\tau, S, 0)-\lambda \frac{(\mu-r)}{K_{2}} \widetilde{\mathrm{I}}(\tau, S, 0) .
\end{aligned}
$$

5.2. Discretization. To solve (38), we use a finite difference scheme with the following nation and approximations:

$$
\tau: 0 \leq \cdots n \Delta t \cdots \leq(N-1) \Delta t, \quad n=0,1, \ldots, N-1,
$$

$S: 0 \leq \cdots i \Delta X \cdots \leq(I-1) \Delta X=S_{\max }, \quad i=0,1, \ldots, I-1$,

$V: 0 \leq \cdots j \Delta V \cdots \leq J \Delta V=V_{\max }, \quad j=0,1, \ldots, J-1$,

$$
\begin{gathered}
U_{i, j}^{n}=\widetilde{C}(n \Delta t, i \Delta X, j \Delta V), \\
\mathrm{I}_{i, j}^{n}=\widetilde{\mathrm{I}}(n \Delta t, i \Delta X, j \Delta V), \\
\mathrm{II}_{i, j}^{n}=\widetilde{\mathrm{II}}(n \Delta t, i \Delta X, j \Delta V), \\
\frac{\partial \widetilde{C}}{\partial \tau}(\tau, S, V) \approx \frac{U_{i, j}^{n+1}-U_{i, j}^{n}}{\Delta t}, \\
\frac{\partial \widetilde{C}}{\partial S}(\tau, S, V) \approx \frac{U_{i+1, j}^{n}-U_{i-1, j}^{n}}{2 \Delta X}, \\
\frac{\partial^{2} \widetilde{C}}{\partial S \partial V}=\frac{(\tau, S, V) \approx \frac{U_{i+1, j}^{n}-2 U_{i, j}^{n}+U_{i-1, j}^{n}}{(\Delta X)^{2}},}{\frac{U_{i+1, j+1}^{n}+U_{i-1, j-1}^{n}-U_{i-1, j+1}^{n}-U_{i+1, j-1}^{n}}{4 \Delta X \Delta V}} \\
\frac{\partial \widetilde{C}}{\partial V}(\tau, S, V) \approx \frac{U_{i, j+1}^{n}-U_{i, j-1}^{n}}{2 \Delta V}, \\
\widetilde{\partial S}, S, V) \approx \frac{U_{i, j+1}^{n}-2 U_{i, j}^{n}+U_{i, j-1}^{n}}{(\Delta V)^{2}} .
\end{gathered}
$$

Now, we evaluate the integral term. First, by performing a change of variable $z:=S \cdot y, y=z / S$ and $d y=d z / S$, the $\widetilde{\mathrm{I}}\left(\tau, S_{t}, V_{t}\right)$ and $\widetilde{\mathrm{II}}\left(\tau, S_{t}, V_{t}\right)$ can be discretized by using the linear interpolation. Thus,

$$
\begin{aligned}
z: 0 \leq \cdots & k \Delta X \cdots(I-2) \Delta X, \quad k=0,1, \ldots, I-2, \\
\widetilde{\mathrm{I}} & =\int_{R_{0}} \frac{\widetilde{C}(\tau, z, V) f(z / S)}{S d z} \\
& \approx \int_{0}^{\infty} \frac{\widetilde{C}(n \Delta t, z, j \Delta V) f(z / i \Delta X)}{(i \Delta X) d z}
\end{aligned}
$$

$$
\begin{aligned}
\widetilde{\mathrm{II}} & =\int_{R_{0}} \frac{\widetilde{C}(\tau, z, V)(z / S-1) f(z / S)}{S d z} \\
& \approx \int_{0}^{\infty} \frac{\widetilde{C}(n \Delta t, z, j \Delta V)(z /(i \Delta X)-1) f(z / i \Delta X)}{(i \Delta X) d z} .
\end{aligned}
$$

Now, by using linear interpolation, we get approximation the following:

$$
\begin{gathered}
\mathrm{I}_{i, j}^{n} \approx A_{i, j}^{n}=\sum_{k=0}^{I-2} \widetilde{A}_{i, j}^{n, k}, \\
\mathrm{II}_{i, j}^{n} \approx B_{i, j}^{n}=\sum_{k=0}^{I-2} \widetilde{B}_{i, j}^{n, k},
\end{gathered}
$$

where

$$
\begin{aligned}
& \widetilde{A}_{i, j}^{n, k}=\int_{k \Delta X}^{(k+1) \Delta X} \frac{\widetilde{C}(n \Delta t, z, j \Delta V) f(z / i \Delta X)}{(i \Delta X) d z} \\
& =\int_{k \Delta X}^{(k+1) \Delta X} \frac{(k+1) \Delta X-z}{\Delta X} \\
& \times \frac{\widetilde{C}(n \Delta t, k \Delta X, j \Delta V) f(z / i \Delta X)}{(i \Delta X) d z} \\
& +\int_{k \Delta X}^{(k+1) \Delta X} \frac{z-k \Delta X}{\Delta X} \\
& \times \frac{\widetilde{C}(n \Delta t,(k+1) \Delta X, j \Delta V) f(z / i \Delta X)}{(i \Delta X) d z} \\
& =\frac{1}{2}[k \widetilde{C}(n \Delta t,(k+1) \Delta X, j \Delta V) \\
& -(k+1) \widetilde{C}(n \Delta t, k \Delta X, j \Delta V)] \\
& \cdot\left[\operatorname{erf}\left(\frac{(\gamma-\ln ((k+1) / i))}{\sqrt{2} \delta}\right)\right. \\
& \left.-\operatorname{erf}\left(\frac{(\gamma-\ln (k / i))}{\sqrt{2} \delta}\right)\right] \\
& +\frac{1}{2} i e^{\delta^{2} / 2+\gamma}[\widetilde{C}(n \Delta t, k \Delta X, j \Delta V) \\
& -\widetilde{C}(n \Delta t,(k+1) \Delta X, j \Delta V)] \\
& \cdot\left[\operatorname{erf}\left(\frac{\left(\gamma-\ln ((k+1) / i)+\delta^{2}\right)}{\sqrt{2} \delta}\right)\right. \\
& \left.-\operatorname{erf}\left(\frac{\left(\gamma-\ln (k / i)+\delta^{2}\right)}{\sqrt{2} \delta}\right)\right]
\end{aligned}
$$


Denote the following:

$$
\begin{aligned}
E_{i, j}^{n, k}= & \frac{1}{2}\left[k U_{k+1, j}^{n}-(k+1) U_{k, j}^{n}\right], \\
F_{i, j}^{n, k}= & \frac{1}{2}\left[U_{k, j}^{n}-U_{k+1, j}^{n}\right], \\
G_{i}^{k}= & {\left[\operatorname{erf}\left(\frac{(\gamma-\ln ((k+1) / i))}{\sqrt{2} \delta}\right)\right.} \\
& \left.-\operatorname{erf}\left(\frac{(\gamma-\ln (k / i))}{\sqrt{2} \delta}\right)\right], \\
H_{i}^{k}= & {\left[\operatorname{erf}\left(\frac{\left(\gamma-\ln ((k+1) / i)+\delta^{2}\right)}{\sqrt{2} \delta}\right)\right.} \\
& \left.-\operatorname{erf}\left(\frac{\left(\gamma-\ln (k / i)+\delta^{2}\right)}{\sqrt{2} \delta}\right)\right] .
\end{aligned}
$$

Then, we have $A_{i, j}^{n, k}=E_{i, j}^{n, k} * G_{i}^{k}+i e^{\left(\delta^{2} / 2\right)+\gamma} F_{i, j}^{n, k} * H_{i}^{k}$

$$
\begin{aligned}
& \widetilde{B}_{i, j}^{n, k} \\
& =\int_{k \Delta X}^{(k+1) \Delta X} \frac{\widetilde{C}(n \Delta t, z, j \Delta V)(z /(i \Delta X)-1) f(z /(i \Delta X)-1)}{(i \Delta X) d z} \\
& =\int_{k \Delta X}^{(k+1) \Delta X} \frac{(k+1) \Delta X-z}{\Delta X} \\
& \times \widetilde{C}(n \Delta t, k \Delta X, j \Delta V) \\
& \times\left(\frac{z}{i \Delta X}-1\right) \frac{1}{z \delta \sqrt{2 \pi}} \\
& \times \exp \left(-\frac{(\ln (z / i \Delta X)-\gamma)^{2}}{2 \delta^{2}}\right) d z \\
& +\int_{k \Delta X}^{(k+1) \Delta X} \frac{z-k \Delta X}{\Delta X} \\
& \times \widetilde{C}(n \Delta t,(k+1) \Delta X, j \Delta V)\left(\frac{z}{i \Delta X}-1\right) \\
& \times \frac{1}{z \delta \sqrt{2 \pi}} \exp \left(-\frac{(\ln (z / i \Delta X)-\gamma)^{2}}{2 \delta^{2}}\right) d z \\
& =\frac{1}{2} e^{\left(\delta^{2} / 2\right)+\gamma}[k \widetilde{C}(n \Delta t,(k+1) \Delta X, j \Delta V) \\
& -(k+1) \widetilde{C}(n \Delta t, k \Delta X, j \Delta V)] \\
& {\left[\operatorname{erf}\left(\frac{\left(\gamma-\ln ((k+1) / i)+\delta^{2}\right)}{\sqrt{2} \delta}\right)\right.} \\
& \left.-\operatorname{erf}\left(\frac{\left(\gamma-\ln (k / i)+\delta^{2}\right)}{\sqrt{2} \delta}\right)\right] \\
& +\frac{1}{2 i \sqrt{1-2 \delta^{2}}} e^{\gamma^{2} /\left(1-2 \delta^{2}\right)}
\end{aligned}
$$

$\times[\widetilde{C}(n \Delta t, k \Delta X, j \Delta V)$

$$
\begin{gathered}
-\widetilde{C}(n \Delta t,(k+1) \Delta X, j \Delta V)] \\
\cdot\left[\operatorname { e r f } \left(\sqrt{1-2 \delta^{2}} \frac{(\gamma-\ln ((k+1) / i))}{\sqrt{2} \delta}\right.\right. \\
\left.-\frac{\sqrt{2} \delta \gamma}{\sqrt{1-2 \delta^{2}}}\right) \\
-\operatorname{erf}\left(\sqrt{1-2 \delta^{2}} \frac{(\gamma-\ln (k / i))}{\sqrt{2} \delta}\right. \\
\left.\left.-\frac{\sqrt{2} \delta \gamma}{\sqrt{1-2 \delta^{2}}}\right)\right]-\widetilde{A}_{i, j}^{n, k} .
\end{gathered}
$$

Denote the following:

$$
\begin{gathered}
L_{i}^{k}=\left[\operatorname { e r f } \left(\sqrt{1-2 \delta^{2}} \frac{(\gamma-\ln ((k+1) / i))}{\sqrt{2} \delta}\right.\right. \\
\left.-\frac{\sqrt{2} \delta \gamma}{\sqrt{1-2 \delta^{2}}}\right) \\
-\operatorname{erf}\left(\sqrt{1-2 \delta^{2}} \frac{(\gamma-\ln (k / i))}{\sqrt{2} \delta}\right. \\
\left.\left.-\frac{\sqrt{2} \delta \gamma}{\sqrt{1-2 \delta^{2}}}\right)\right] .
\end{gathered}
$$

Then, we have $B_{i, j}^{n, k}=e^{\left(\delta^{2} / 2\right)+\gamma} E_{i, j}^{n, k} * H_{i}^{k}+\left(1 / i \sqrt{1-2 \delta^{2}}\right)$ $e^{\gamma^{2} /\left(1-2 \delta^{2}\right)} F_{i, j}^{n, k} * L_{i}^{k}-A_{i, j}^{n, k}$, where $\operatorname{erf}(\cdot)$ is the error function defined by $\operatorname{erf}(x)=(2 / \sqrt{\pi}) \int_{0}^{x} e^{-y^{2}} d y$ with $\operatorname{erf}(\infty)=1$ and $\operatorname{erf}(-x)=-\operatorname{erf}(x)$.

Then,

$$
A_{i, j}^{n}=\sum_{k=0}^{I-2} \widetilde{A}_{i, j}^{n, k}, \quad B_{i, j}^{n}=\sum_{k=0}^{I-2} \widetilde{B}_{i, j}^{n, k} .
$$

Finally, discretization of (27) gives

$$
\begin{aligned}
U_{i, j}^{n+1}=- & -1+\Delta t\left(r+K_{0}-\frac{\mu-r}{j \Delta V+K_{2}} K_{1}\right) \\
& \left.+\Delta t j \Delta V i^{2}+\frac{\Delta t j \sigma^{2}}{\Delta V}\right] U_{i, j}^{n} \\
+ & {\left[\frac{\Delta t i}{2}\left(r-K_{1}+\frac{\mu-r}{j \Delta V+K_{2}} K_{2}\right)+\frac{\Delta t j \Delta V i^{2}}{2}\right] U_{i+1, j}^{n} } \\
- & {\left[\frac{\Delta t i}{2}\left(r-K_{1}+\frac{\mu-r}{j \Delta V+K_{2}} K_{2}\right)-\frac{\Delta t j \Delta V i^{2}}{2}\right] U_{i-1, j}^{n} } \\
+ & {\left[\frac{\Delta t}{2 \Delta V}\left(\kappa(\varphi-j \Delta V)-\rho \sigma \frac{\mu-r}{j \Delta V+K_{2}} j \Delta V\right)\right.} \\
& \left.+\frac{\Delta t j \sigma^{2}}{2 \Delta V}\right] U_{i, j+1}^{n}
\end{aligned}
$$




$$
\begin{aligned}
& -\left[\frac{\Delta t}{2 \Delta V}\left(\kappa(\varphi-j \Delta V)-\rho \sigma \frac{\mu-r}{j \Delta V+K_{2}} j \Delta V\right)\right. \\
& \left.-\frac{\Delta t j \sigma^{2}}{2 \Delta V}\right] U_{i, j-1}^{n} \\
& +\left[\frac{\Delta t i j \sigma \rho}{4}\right] U_{i+1, j+1}^{n}+\left[\frac{\Delta t i j \sigma \rho}{4}\right] U_{i-1, j-1}^{n} \\
& -\left[\frac{\Delta t i j \sigma \rho}{4}\right] U_{i-1, j+1}^{n}-\left[\frac{\Delta t i j \sigma \rho}{4}\right] U_{i+1, j-1}^{n} \\
& +\lambda \Delta t A_{i, j}^{n}-\frac{\lambda \Delta t(\mu-r)}{j \Delta V+K_{2}} B_{i, j}^{n} .
\end{aligned}
$$

It equals

$$
\begin{aligned}
U_{i, j}^{n+1}= & c U_{i, j}^{n}+d U_{i+1, j}^{n}+e U_{i-1, j}^{n}+g U_{i, j+1}^{n}+h U_{i, j-1}^{n} \\
& +q\left(U_{i+1, j+1}^{n}+U_{i-1, j-1}^{n}-U_{i-1, j+1}^{n}-U_{i+1, j-1}^{n}\right) \\
& +a A_{i, j}^{n}+b B_{i, j}^{n},
\end{aligned}
$$

where

$$
\begin{aligned}
c(i, j)= & 1-\Delta t\left(r+K_{0}-\frac{\mu-r}{j \Delta V+K_{2}} K_{1}\right) \\
& -\Delta t j \Delta V i^{2}-\frac{\Delta t j \sigma^{2}}{\Delta V}, \\
d(i, j)= & \frac{\Delta t i}{2}\left(r-K_{1}+\frac{\mu-r}{j \Delta V+K_{2}} K_{2}\right)+\frac{\Delta t j \Delta V i^{2}}{2}, \\
e(i, j)= & -\frac{\Delta t i}{2}\left(r-K_{1}+\frac{\mu-r}{j \Delta V+K_{2}} K_{2}\right)+\frac{\Delta t j \Delta V i^{2}}{2}, \\
g(i, j)= & \frac{\Delta t}{2 \Delta V}\left(\kappa(\varphi-j \Delta V)-\rho \sigma \frac{\mu-r}{j \Delta V+K_{2}} j \Delta V\right)+\frac{\Delta t j \sigma^{2}}{2 \Delta V}, \\
h(i, j)- & \frac{\Delta t}{2 \Delta V}\left(\kappa(\varphi-j \Delta V)-\rho \sigma \frac{\mu-r}{j \Delta V+K_{2}} j \Delta V\right)+\frac{\Delta t j \sigma^{2}}{2 \Delta V}, \\
q(i, j)= & \frac{\Delta t i j \sigma \rho}{4}, \quad a(i, j)=\lambda \Delta t, \\
b(i, j)= & -\frac{\lambda \Delta t(\mu-r)}{j \Delta V+K_{2}} .
\end{aligned}
$$

\section{Numerical Results}

In this section, we adopted the basic set of appropriate parameter values of the model shown in Table 1 as our basic set of parameter values to solve the finite difference equation (38).

Figure 1 shows the payoff with the price and the volatility. The solution of (38) is shown in Figure 2. All numerical experiments have been implemented by MATLAB R2011b software on a $2.0-\mathrm{GHz}$ Intel Core PC.
TABLE 1: Basic set of appropriate parameter values of the model.

\begin{tabular}{lc}
\hline$\mu$ & 0.23 \\
$\kappa$ & 3.46 \\
$\varphi$ & $(0.0894)^{2}$ \\
$\delta$ & 0.0001 \\
$\rho$ & -0.82 \\
$T$ & 1 \\
$S_{\max }$ & 200 \\
$V_{\max }$ & 1 \\
$r$ & 0.039 \\
$\sigma$ & 0.14 \\
$\lambda$ & 0.47 \\
$\gamma$ & -0.086 \\
$K$ & 100 \\
$N$ & 2000 \\
$I$ & 30 \\
$J$ & 30 \\
\hline
\end{tabular}

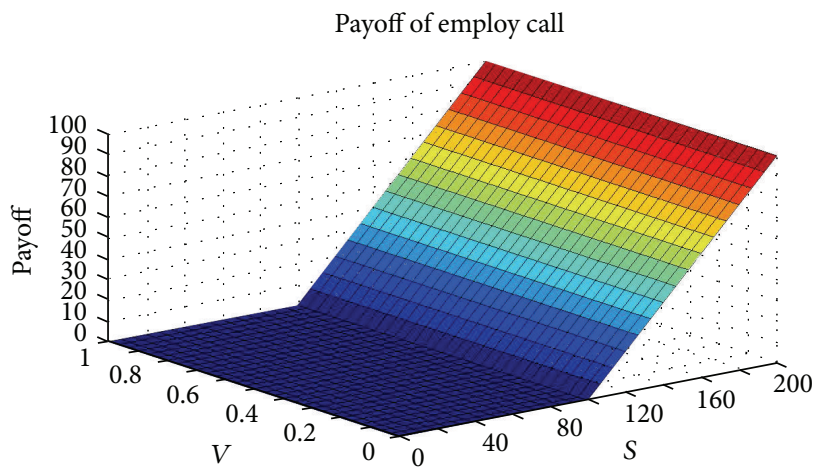

FIGURE 1: The payoff with the price and the volatility.

TABLE 2: European option prices.

\begin{tabular}{lc}
\hline Price & $I$ \\
\hline 8.7858 & 10 \\
9.3014 & 20 \\
9.6929 & 30 \\
9.7006 & 40 \\
\hline
\end{tabular}

Now, we suppose that the underlying stock price process follows (2) and there is a European call option with $S_{0}=$ 100, $\sqrt{V_{0}}=0.35$, and, other parameters in Table 1 . Table 2 shows the European option prices solved by FDM with different $I$ which stands for the different accuracy.

\section{Conclusion}

With risk-minimization criterion, we employ the minimal martingale measure to solve the pricing problem in an incomplete market. Then, we obtain the Radon-Nikodym derivative under the minimal martingale measure and a 


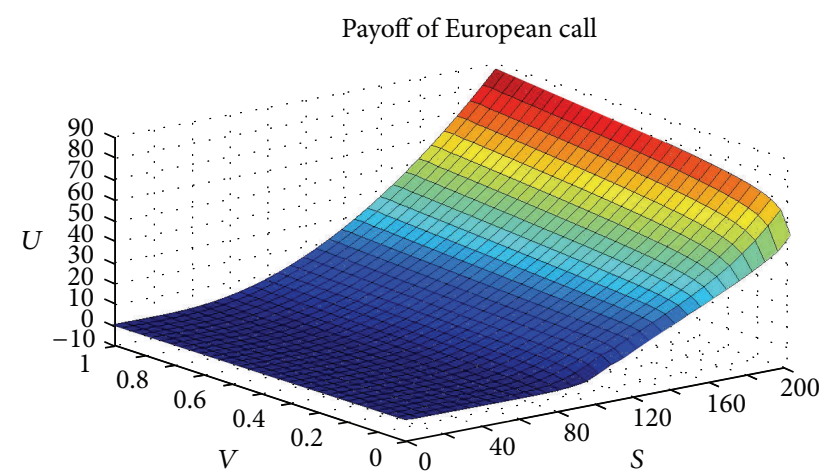

Figure 2: The solution of (38).

partial integro-differential equation (PIDE) for the European option. Since it is difficult to get the exact solution of PIDE in our model, a FDM scheme is proposed to compute the solution approximately. Finally, we complete a European call option price, and it is shown that our method is stable and locally accurate.

\section{References}

[1] F. Black and M. Scholes, "The pricing of options and corporate liabilities," Journal of Political Economy, vol. 81, no. 3, pp. 637659, 1973.

[2] J. Hull and A. White, "The pricing of options on assets with stochastic volatilities," The Journal of Finance, vol. 42, no. 2, pp. 281-300, 1987.

[3] L. Scott, "Option pricing when the variance changes randomly: theory, estimation, and an application," The Journal of Financial and Quantitative Analysis, vol. 22, no. 4, pp. 419-438, 1987.

[4] J. Wiggins, "Option values under stochastic volatility: theory and empirical estimates," Journal of Financial Economics, vol. 19, no. 2, pp. 351-372, 1987.

[5] S. Melino and S. M. Turnbull, "Pricing foreign currency options with stochastic volatility," Journal of Econometrics, vol. 45, no. 12, pp. 239-265, 1990.

[6] S. Melino and S. M. Turnbull, "Misspecification and the pricing and hedging of long-term foreign currency options," Journal of International Money and Finance, vol. 14, no. 3, pp. 373-393, 1995.

[7] E. M. Stein and J. C. Stein, "Stock price distributions with stochastic volatility: an analytic approach," The Review of Financial Studies, vol. 4, no. 4, pp. 727-752, 1991.

[8] S. Heston, "A closed-form solution for options with stochastic volatility with applications to bond and currency options," The Review of Financial Studies, vol. 6, no. 2, pp. 327-343, 1993.

[9] R. Merton, "Option pricing when underlying stock returns are discontinuous," Journal of Financial Economics, vol. 3, no. 1-2, pp. 125-144, 1976.

[10] G. Bakshi, C. Cao, and Z. Chen, "Empirical performance of alternative option pricing models," The Journal of Finance, vol. 52, no. 5, pp. 2003-2049, 1997.

[11] F. E. Benth and T. Meyer-Brandis, "The density process of the minimal entropy martingale measure in a stochastic volatility model with jumps," Finance and Stochastics, vol. 9, no. 4, pp. 563-575, 2005.

[12] D. Bates, "The crash of '87: was it expected? The evidence from options markets," The Journal of Finance, vol. 46, no. 3, pp. 10091044, 1991.

[13] H. Föllmer and P. Leukert, "Efficient hedging: cost versus shortfall risk," Finance and Stochastics, vol. 4, no. 2, pp. 117-146, 2000.

[14] J. Kallsen, "A utility maximization approach to hedging in incomplete markets," Mathematical Methods of Operations Research, vol. 50, no. 2, pp. 321-338, 1999, Financial optimization.

[15] J. Cvitanić, W. Schachermayer, and H. Wang, "Utility maximization in incomplete markets with random endowment," Finance and Stochastics, vol. 5, no. 2, pp. 259-272, 2001.

[16] T. Bielecki and M. Jeanblanc, Indifference Prices in Indifference Pricing, Theory and Applications, Financial Engineering, Edited by R. Carmona, Princeton University Press, 2008.

[17] M. Schweizer, "Option hedging for semimartingales," Stochastic Processes and their Applications, vol. 37, no. 2, pp. 339-363, 1991.

[18] H. F. Föllmer and M. Schweizer, "Hedging of contingent claims under incomplete information," in Applied Stochastic Analysis, M. H. A. Davis and R. J. Elliott, Eds., vol. 5, pp. 389-414, Stochastics Monographs, London, UK, 1991.

[19] C. Chiarella and A. Ziogas, "American call options under jumpdiffusion processes: a fourier transform approach," Applied Mathematical Finance, vol. 16, no. 1-2, pp. 37-79, 2009.

[20] A. M. Matache, C. Schwab, and T. P. Wihler, "Fast numerical solution of parabolic integrodifferential equations with applications in finance," SIAM Journal on Scientific Computing, vol. 27, no. 2, pp. 369-393, 2005.

[21] G. H. Meyer, "The numerical valuation of options with underlying jumps," Acta Mathematica, vol. 67, no. 1, pp. 69-82, 1998.

[22] P. Carr and A. Hirsa, "Why be backward? forward equations for American options," Risk, vol. 16, no. 1, pp. 103-107, 2003.

[23] Y. d'Halluin, P. A. Forsyth, and G. Labahn, "A penalty method for American options with jump diffusion processes," Numerische Mathematik, vol. 97, no. 2, pp. 321-352, 2004.

[24] M. Briani, C. La Chioma, and R. Natalini, "Convergence of numerical schemes for viscosity solutions to integro-differential degenerate parabolic problems arising in financial theory," Numerische Mathematik, vol. 98, no. 4, pp. 607-646, 2004. 


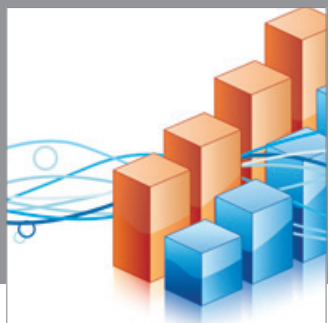

Advances in

Operations Research

mansans

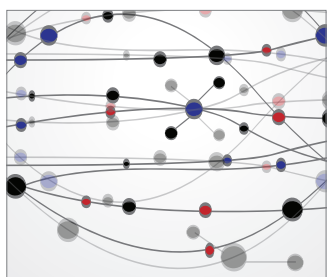

The Scientific World Journal
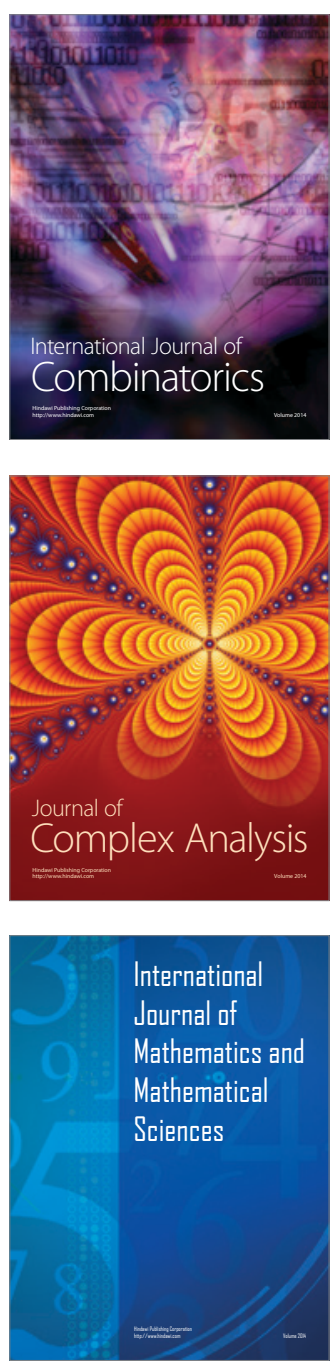
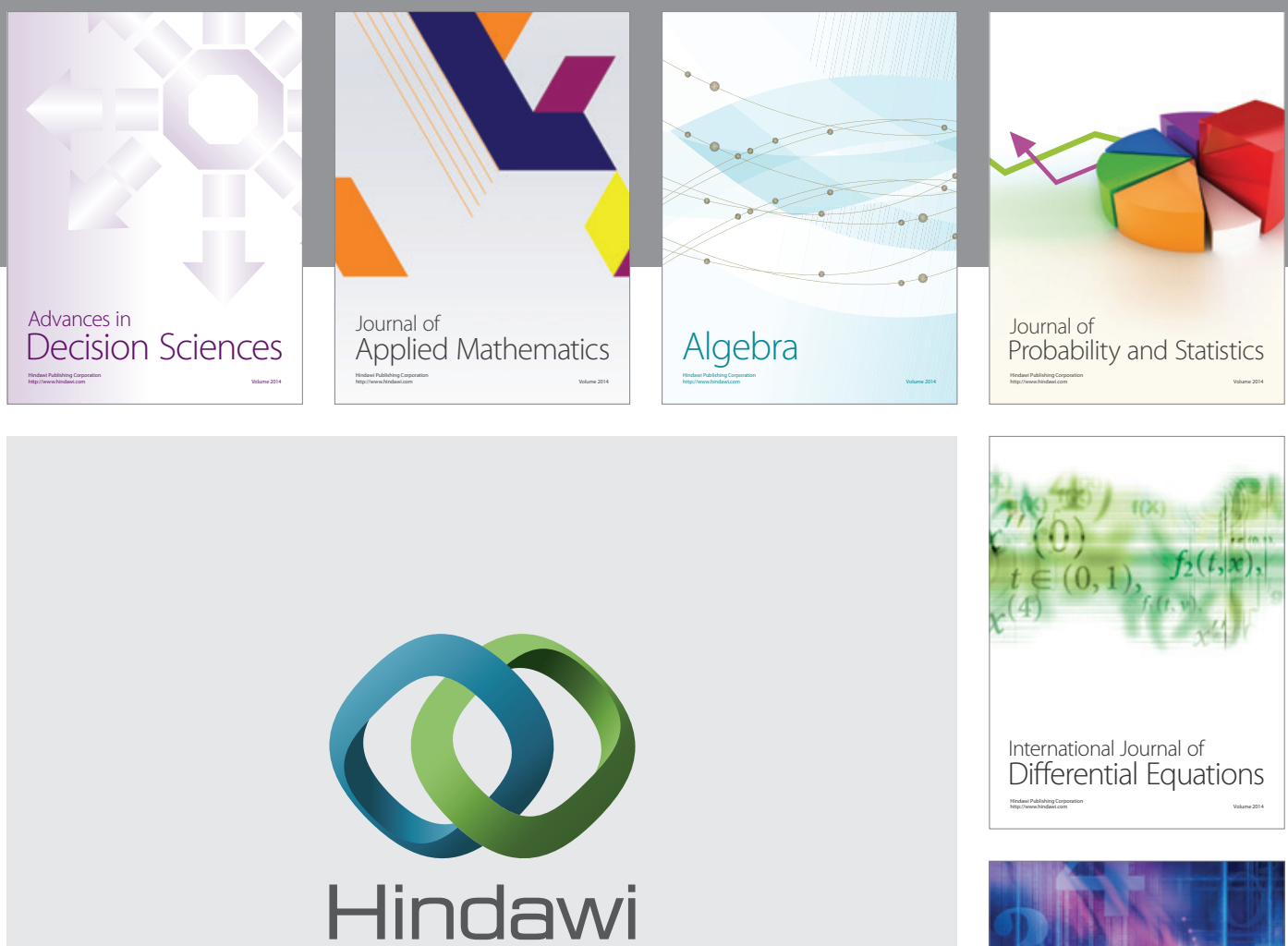

Submit your manuscripts at http://www.hindawi.com
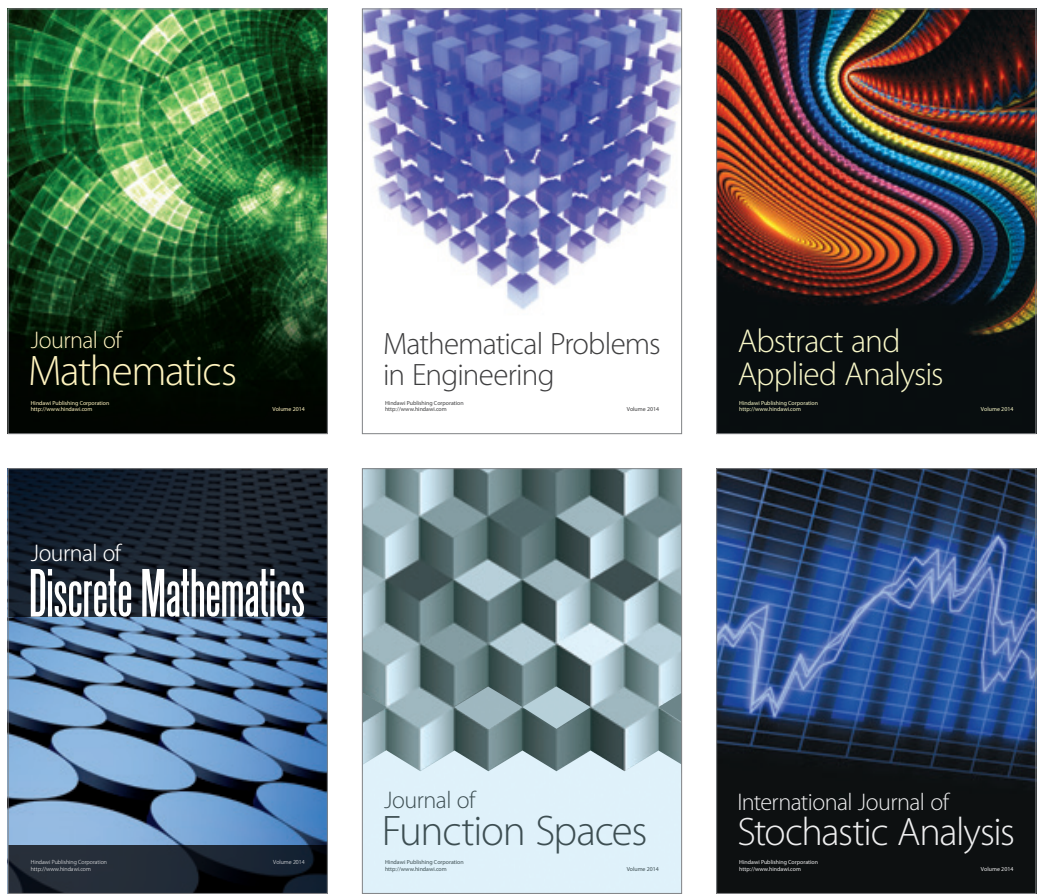

Journal of

Function Spaces

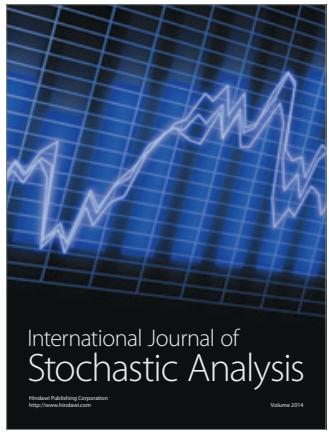

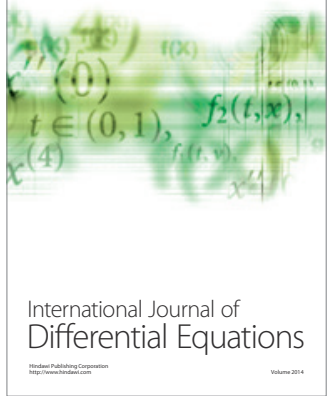
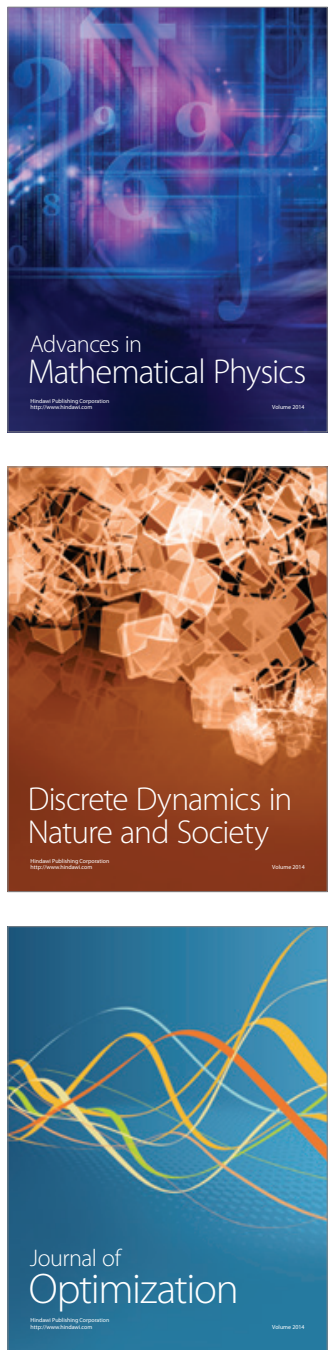